A0017 Opioid-Free Anesthesia with Neuromonitoring in Acoustic Neuroma Surgery: Case Report

Sandra Fernandez-Caballero, ${ }^{1}$ David Velasco-Villanueva ${ }^{1}$

${ }^{1}$ Department of Anesthesiology, Hospital Clínico Universitario de Valladolid, Valladolid, Spain

Background: Perioperative opioid administration is associated with adverse effects, particularly in neuroanesthesia. The opioid-free anesthesia (OFA) with propofol, ketamine, and scalp blockade helps to prevent those side-effects and eases neuromonitoring during the intervention.

Case Description: A 59-year-old woman presented with a history of unilateral hearing loss, muscle cramps in the neck, and balance disturbances over the past few months. The magnetic resonance imaging (MRI) revealed a right cerebellopontine-angle lesion compatible with an acoustic neuroma. The patient was scheduled to undergo elective surgery (retromastoid suboccipital craniectomy and excision of the tumor) general anesthesia. Standard monitors included were electrocardiogram (ECG), noninvasive and continuous blood pressure, pulse oximeter, capnograph, brain function monitor (Sedline), and electrophysiological monitoring (somatosensory evoked potentials, motor evoked potentials, electromyography); and use of intraoperative neuromuscular blockade (NMB) was avoided.

General anesthesia was induced in the standard way, a scalp block was performed, and it was maintained with a continuous infusion of propofol and ketamine throughout the procedure, which lasted approximately 12 hours. During the procedure, some disturbances in the electrophysiological recording of the right facial nerve were reported. After the intervention, she was admitted to the PICU and had good control of the pain during the postoperative period, with a visual analog scale (VAS) score of 2 , requiring just $2 \mathrm{mg}$ of morphine. The patient was discharged to the ward the next day.

Conclusions: The use of OFA during neurosurgery offers significant advantages, including improvement of hemodynamic stability, adequate hypnotic state, and maintenance of a stable plane of anesthesia. In this case, OFA with propofol and ketamine combined with scalp block provided good pain relief without altering the electrophysiological recording.

\section{A0018 Anesthetic Management of Children with Craniosynostosis for Corrective Surgery: A 10-Year Experience \\ Karen R. Lionel, ${ }^{1}$ Arunkumar Natarajan, ${ }^{1}$ Ramamani Mariap- pan, ${ }^{1}$ Georgene Singh, ${ }^{1}$ Ranjith K. Moorthy ${ }^{2}$ \\ ${ }^{1}$ Department of Anaesthesia, Christian Medical College, Vellore, India \\ ${ }^{2}$ Department of Neurological Sciences, Christian Medical College, Vellore, India}

Background: Craniosynostosis is a developmental defect characterized by premature fusion of one or more skull sutures leading to deformity and restricted growth of brain. It is associated with different syndromes and compounded by the various inherent intricacies of infant physiology making it a challenge for the neuroanesthesiologist.
Materials and Methods: Perioperative data of infants and children who had undergone craniosynostosis correction surgery by a single surgical team over a period of 10 years were retrospectively collected after IRB approval.

Results: There were 22 patients, of whom 9 (40.9\%) were females; Mean age-21.4 months; weight of $8.6 \mathrm{~kg}$. The most common suture involved was coronal in 18 (81.8\%), followed by sagittal 13 (59.1\%), metopic in 12 (54.6\%), and lambdoid in 11 (50\%). Seven (31.8\%) infants had all four-suture involvement, two had three sutures, seven had two sutures, and six had single-suture involvement. Of these, 13 (59.1\%) were syndromic (Crouzon's, Apert's, and Down's syndromes).

Sevoflurane induction was performed in 17 (77.3\%), and rest had intravenous induction. Anesthesia was maintained with inhalational in 18 (81.8\%), and 4 (18.2\%) had combination of IV and inhalational agents. Eighteen (81.8\%) had an anticipated difficult airway; of these, 5 had CL grade of 3 , most of them (4/5) were syndromic.

Average blood loss was $40.9 \mathrm{~mL} / \mathrm{kg}$; syndromic group had higher loss $51.2 \mathrm{~mL} / \mathrm{kg}$ vs. $25.9 \mathrm{~mL} / \mathrm{kg}(p=0.049)$. Three out of 22 patients did not receive tranexamic acid, these children had increased blood loss 68.3 vs. $36.5 \mathrm{~mL} / \mathrm{kg}(p=0.09$ ). Hypofibrinogenemia was the most common coagulation abnormality. Those who had intraoperative coagulation abnormality had higher blood loss, $58.0 \mathrm{~mL} / \mathrm{kg}$ vs. $29.7 \mathrm{~mL} / \mathrm{kg}$ $(p=0.004) .14 / 22$ (64\%) had intraoperative hypotension requiring nor-adrenaline infusion. Few (2/22) had both noradrenaline and adrenaline. Children who had intraoperative ABG (15/22), six (40\%) had lactate of > 2 mmol/L. Hyperchloremia (45.4\%) was the most commonly observed electrolyte abnormality, followed by hypocalcemia. Average duration of anesthesia was 352 minutes. There was no correlation between the number of sutures involved and the duration of surgery $(p=0.418)$ nor with the blood loss $(p=0.331)$.

Four (18\%) out of 22 children had postoperative ventilation. The mean ICU and hospital stays were 1.7 and 5 days, respectively. Seven out of 22 had postoperative coagulation profile, of whom 1 had both low levels of fibrinogen and a prolonged APTT and 4 had purely hypofibrinogenemia; 1 had thrombocytopenia. No postoperative complication or death noted in these series.

Conclusions: Anticipation, adequate preparation for airway and blood loss, administration of titrated anesthetic, maintenance of hemodynamics, and timely administration of tranexamic acid and blood and blood products reduced the complication in these children.

\section{A0019 Requirement of Propofol for Induction of} Anesthesia in Patients with Traumatic Brain Injury Using Bilateral Bi-spectral Index

Seham Syeda, ${ }^{1}$ V. Bhadri Narayan, ${ }^{1}$ Sonia Bansal ${ }^{1}$

${ }^{1}$ Department of Neuroanaesthesia, National Institute of Mental Health and Neurosciences, Bengaluru, Karnataka, India

Background: Studies have demonstrated a decreased requirement of propofol for induction of anesthesia in patients undergoing neurosurgical procedures as compared with non-neurological procedures. There is a paucity of 
literature with regard to the anesthetic requirement in patients with traumatic brain injury (TBI). The aim of our study was to determine the dose of propofol required for induction of anesthesia in patients with different grades of TBI undergoing surgery.

Materials and Methods: This prospective, observational study included patients with mild-, moderate-, and severe-grade TBI undergoing surgery within 48 hours of injury. Baseline bi-spectral index (BIS) values were recorded on injured and normal sides using bilateral BIS sensor. Anesthesia was induced with "a constant rate infusion of propofol using a target-controlled infusion (TCI) pump." Once the BIS value reached $\leq 40$, plasma site (Cp) and effect site (Ce) concentration and total dose of propofol required were noted from the TCI pump. Bilateral BIS and GCS at the end of surgery and GCS at discharge were also noted.

Results: Of the 96 patients, 27, 36, and 33 patients belonged to mild, moderate, and severe head injury, respectively. Baseline BIS on the injured side were 80,71 , and 55 , and on uninjured side were 89,81 , and 65 in mild, moderate and severe head injury. This difference between normal and injured side was statistically significant $(p<0.001)$ in each group. The Ce of propofol at induction in mild, moderate, and severe head injury were $6 \pm 0.9,5.82 \pm 0.98$, and $4.47 \pm 1.4 \mu \mathrm{g} / \mathrm{mL}(p<0.001)$, and dose of propofol required was $1.9 \pm 0.2,1.8 \pm 0.4$, and $1.41 \pm 0.5 \mathrm{mg} / \mathrm{kg}$, respectively $(p<0.001)$.

Conclusions: BIS values at baseline were significantly different between the groups on both sides. Dose of propofol required for induction of anesthesia was significantly lower in patients with severe head injury when compared with mild and moderate head injury.

\section{A0020 An Unusual Case of Acute Trigeminal Neuralgia Attack Precipitating Acute Heart Failure and Subsequent Anesthetic Management \\ Rajesha Srinivasaiah, ${ }^{1}$ Anshul Yadav, ${ }^{1}$ Shehla Shakooh, ${ }^{1}$ \\ Sandeep Lakhani ${ }^{1}$ \\ ${ }^{1}$ The Walton Centre NHS Foundation Trust, Liverpool, United Kingdom}

Background: Trigeminal neuralgia is a neuropathic pain disorder characterized by episodes of paroxysmal electric shock-like pain in the distribution of one or more divisions of trigeminal nerve. Cardiac symptoms like syncope associated with trigeminal neuralgia have been reported before. We report a case of patient whose acute trigeminal neuralgia attack precipitated an acute heart failure.

Case Description: An 82-year-old woman was posted for microvascular decompression (MVD) of trigeminal nerve for trigeminal neuralgia. Her past medical history included hypertension and mild mitral regurgitation. The preoperative investigations, including 2D echo, were essentially normal. Two days before the surgery, patient developed acute exacerbation of trigeminal neuralgia and within 2 hours developed breathlessness which was due to an acute left ventricular failure. Echo revealed LVEF of 34\% with severe systolic dysfunction and severe MR. She was appropriately treated in CCU. Later she was reviewed by a neurosurgeon and anesthetist.
The decision was taken to change her treatment from MVD to percutaneous balloon compression (PBC) of TGN.

$\mathrm{PBC}$ involves cannulation of the foramen ovale, followed by inflation of a Fogarty balloon in Meckel's cave under fluoroscopic guidance to damage retrogasserian trigeminal fibers. It is a short-duration, minimally invasive procedure done as a day care case under GA. Trigeminal neuralgia attacks are amongst the most painful conditions known. Acute pain can cause activation of sympathetic nervous system and increased catecholamine levels resulting in increased cardiac work. It also causes activation of the renin-angiotensin-aldosterone system leading to fluid retention and overload.

Conclusions: Cardiac symptoms are very rare manifestations of trigeminal neuralgia. Balloon compression of trigeminal nerve is an important and useful treatment option for patients with trigeminal neuralgia in elderly and frail people when other forms of more invasive treatments are not suitable.

\section{A0021 To Study the Outcome of Massive Transfusion in Pediatric Patients Undergoing Elective Craniotomy: \\ A Retrospective Descriptive Study \\ Swati Patel, ${ }^{1}$ Ramamani Mariappan ${ }^{1}$ \\ 'Department of Anesthesia, Christian Medical College, Vellore, Tamil Nadu, India}

Background: Children undergoing surgical resection of vascular tumor and malformations are at a great risk of rapid, massive blood loss. The primary goals while managing these children who need massive transfusion are avoiding hypotension, maintaining adequate CPP and oxygenation. Goals are achieved by transfusing appropriate volume of blood and blood products at an appropriate time to facilitate faster recovery with minimal complications.

Materials and Methods: Children who received blood transfusion during the elective craniotomy over a period of 5 years were taken from the computerized hospital in-patient data source (CHIPS), of whom, those who had > 50\% volume loss in 3 hours were analyzed. Out of 140 children who received transfusion during elective craniotomy, a total of 26 children received massive transfusion. All charts were analyzed in detail. Patient demographics, preoperative, intraoperative factors were correlated with postoperative factors to predict the morbidity and mortality.

Results: Demographics (median and interquartile range [IQR]): Age 4 (2-12) years; weight $14(11-30) \mathrm{kg}$; height 91 (46-136) cm; BMI 13 (9-17) kg/m², male-to-female ratio $16: 10$. Four out of 26 children had congenital anomalies. Intraoperative parameters: Average preoperative Hb was (median IOR) 10 (8-12) g/dL. 16/26(61\%) received inhalational anesthetic and 10/26 (39\%) received both inhalational and TIVA for maintenance. Regarding the IV access, 25/26 (96\%) of children had two peripheral lines along with one central line. 12/26 (46\%) children received tranexamic acid. Average blood loss was $52.5 \pm 34.5 \mathrm{~mL} / \mathrm{kg}$. 14/26 (53\%) children had intraoperative hemodynamic instability who needed noradrenaline, 9 had no hemodynamic instability, and 1 had 